\title{
Article
}

\section{Impact of 1-Hour Bundle Achievement in Septic Shock}

\author{
Byuk Sung Ko ${ }^{1}$, Sung-Hyuk Choi ${ }^{2} \mathbb{D}$, Tae Gun Shin ${ }^{3}{ }^{\mathbb{D}}$, Kyuseok Kim ${ }^{4}{ }^{(D}$, You Hwan Jo ${ }^{5}$, Seung Mok Ryoo ${ }^{6}$, \\ Yoo Seok Park ${ }^{7}$, Woon Yong Kwon ${ }^{8}$, Han Sung Choi ${ }^{9}$, Sung Phil Chung ${ }^{10}{ }^{(D)}$, Gil Joon Suh ${ }^{8}$, \\ Hyunggoo Kang ${ }^{1} \mathbb{D}$, Tae Ho Lim ${ }^{1,+}$, Donghee Son ${ }^{11} \mathbb{D}$ and Won Young Kim ${ }^{6, *} \mathbb{D}$
}

1 Department of Emergency Medicine, College of Medicine, Hanyang University, Seoul 04763, Korea; postwinston@gmail.com (B.S.K.); emer0905@gmail.com (H.K.); erthim@gmail.com (T.H.L.)

2 Department of Emergency Medicine, Korea University Guro Hospital, Seoul 08308, Korea; kuedchoi@korea.ac.kr

3 Department of Emergency Medicine, Samsung Medical Center, Sungkyunkwan University School of Medicine, 81 Irwon-ro, Gangnam-gu, Seoul 06351, Korea; tackles@naver.com

4 Department of Emergency Medicine, CHA University School of Medicine, CHA Bundang Medical Center, Seongnam-si 13449, Gyeonggi-do, Korea; dreinstein70@gmail.com

5 Department of Emergency Medicine, Seoul National University Bundang Hospital, Seongnam-si 13620, Gyeonggi-do, Korea; drakejo@snubh.org

6 Department of Emergency Medicine, College of Medicine, Asan Medical Center, University of Ulsan, Seoul 05505, Korea; chrisryoo@gmail.com

7 Department of Emergency Medicine, Yonsei University College of Medicine, Seoul 03722, Korea; pys0905@yuhs.ac

8 Department of Emergency Medicine, Seoul National University Hospital, Seoul 03080, Korea; kwy711@hanmail.net (W.Y.K.); suhgil@snu.ac.kr (G.J.S.)

9 Department of Emergency Medicine, Kyung Hee University Hospital, Seoul 02453, Korea; hsg3748@hanmail.net

check for updates

Citation: Ko, B.S.; Choi, S.-H.; Shin, T.G.; Kim, K.; Jo, Y.H.; Ryoo, S.M.; Park, Y.S.; Kwon, W.Y.; Choi, H.S.; Chung, S.P.; et al. Impact of 1-Hour Bundle Achievement in Septic Shock. J. Clin. Med. 2021, 10, 527. https:// doi.org/10.3390/jcm10030527

Academic Editor: Toshiaki Iba Received: 24 December 2020

Accepted: 22 January 2021

Published: 2 February 2021

Publisher's Note: MDPI stays neutral with regard to jurisdictional claims in published maps and institutional affiliations.

Copyright: (c) 2021 by the authors. Licensee MDPI, Basel, Switzerland. This article is an open access article distributed under the terms and conditions of the Creative Commons Attribution (CC BY) license (https:/ / creativecommons.org/licenses/by/ $4.0 /)$.
10 Department of Emergency Medicine, Gangnam Severance Hospital, Seoul 06273, Korea; emstar@naver.com

11 Biostatistical Consulting and Research Lab, Medical Research Collaborating Center, Hanyang University, Seoul 04763, Korea; sdh9123@hanyang.ac.kr

* Correspondence: wonpia73@naver.com

+ Won Young Kim and Tae Ho Lim contributed equally to this work.

\begin{abstract}
This study aimed to address the impact of 1-hr bundle achievement on outcomes in septic shock patients. Secondary analysis of multicenter prospectively collected data on septic shock patients who had undergone protocolized resuscitation bundle therapy at emergency departments was conducted. In-hospital mortality according to 1-h bundle achievement was compared using multivariable logistic regression analysis. Patients were also divided into 3 groups according to the time of bundle achievement and outcomes were compared to examine the difference in outcome for each group over time: group 1 ( $\leq 1 \mathrm{~h}$ reference), group 2 (1-3 h) and group $3(3-6 \mathrm{~h})$. In total, 1612 patients with septic shock were included. The 1-h bundle was achieved in 461 (28.6\%) patients. The group that achieved the 1-h bundle did not show a significant difference in in-hospital mortality compared to the group that did not achieve the 1-h bundle on multivariable logistic regression analysis ( $<1$ vs. $>1 \mathrm{~h}$ ) (odds ratio $=0.74, p=0.091$ ). However, 3 - and 6 - $\mathrm{h}$ bundle achievements showed significantly lower odds ratios of in-hospital mortality compared to the group that did not achieve the bundle ( $<3$ vs. $>3 \mathrm{~h},<6$ vs. $>6 \mathrm{~h}$, odds ratio $=0.604$ and 0.458 , respectively). There was no significant difference in in-hospital mortality over time for group 2 and 3 compared to that of group 1. One-hour bundle achievement was not associated with improved outcomes in septic shock patients. These data suggest that further investigation into the clinical implications of 1-h bundle achievement in patients with septic shock is warranted.
\end{abstract}

Keywords: sepsis; septic shock; 1-h bundle; outcome; mortality; emergency department 


\section{Introduction}

An estimated 48.9 million cases of sepsis have been reported, accounting for $19.7 \%$ of all global deaths $[1,2]$. The incidence of sepsis increases with advanced age, comorbidities, and immunocompromised status [3,4]. This may also be due to increased detection of early sepsis as a result of intensive sepsis education and awareness campaigns. Despite advances in recent critical care, sepsis remains a serious disease with high mortality and morbidity.

Early identification and management is essential in septic patients. In 2005, the Surviving Sepsis Campaign (SSC) suggested a protocolized bundle therapy to facilitate implementation at the bedside with a defined target $[5,6]$. SSC bundles were revised from 6-h to 3-h bundles in 2015. The guidelines published in 2018 state that this resuscitation bundle treatment should be initiated within $1 \mathrm{~h}$ of the emergency department (ED) triage time or the earliest chart annotation if presenting from another care venue, named the $1-\mathrm{h}$ bundle [7]. The 1-h bundle is composed of the following five elements: measuring the lactate level, obtaining blood culture prior to administration of antibiotics, administering broad-spectrum antibiotics, beginning rapid administration of $30 \mathrm{~mL} / \mathrm{kg}$ crystalloid fluid for hypotension or lactate $\geq 4 \mathrm{mmol} / \mathrm{L}$, and administering vasopressors if the patient is hypotensive during or after fluid resuscitation to maintain mean arterial pressure (MAP) at $\geq 65 \mathrm{mmHg}$ within $1 \mathrm{~h}$ from sepsis recognition. However, in October 2019, the definition of time zero changed to the time of shock recognition [8]. This recommendation is based on a study that reported a significant reduction in mortality associated with timely completion of 3-h bundles [9]. Although this study did not address the direct effect of the 1-h bundle, it provided supporting evidence. However, there are concerns regarding the lack of evidence supporting the cut-off of 1-h and a concern about the 1-h bundle causing hasty management decisions, inappropriate fluid administration, and indiscriminate use of broad-spectrum antibiotics $[10,11]$. The quality of evidence supporting the individual elements of the bundle varies from low to moderate [7]. To the best of our knowledge, no study has examined the prognostic value of the 1-h bundle achievement in sepsis and septic shock.

We aimed to evaluate the impact of 1-h bundle achievement in patients with septic shock visiting the ED. We hypothesized that 1-h bundle achievement is not associated with significantly better outcomes than 3- or 6-h bundle achievement.

\section{Methods}

\subsection{Study Design}

Secondary analysis of prospectively collected data was conducted involving $10 \mathrm{Ko}-$ rean university-affiliated hospital EDs using data from the Korean Shock Society septic shock registry from October 2015 to December 2018. Patients aged $\geq 19$ years who met the inclusion criteria (evidence of refractory hypotension or hypoperfusion in patients with suspected or confirmed infection) were included $[12,13]$. Hypotension was defined as systolic blood pressure (SBP) $<90 \mathrm{mmHg}$, $\mathrm{MAP}<70 \mathrm{mmHg}$, or an SBP decrease of $>40 \mathrm{mmHg}$. Refractory hypotension was defined as persistent hypotension despite the administration of fluid challenge ( $30 \mathrm{~mL} / \mathrm{kg}$ of crystalloid fluid) or the requirement of vasopressors to maintain an SBP of $\geq 90 \mathrm{mmHg}$ or a MAP of $\geq 70 \mathrm{mmHg}$. Hypoperfusion was defined as the presence of serum lactate levels $\geq 4 \mathrm{mmol} / \mathrm{L}$. Patients were excluded if they had given "do not attempt resuscitation" orders, met the inclusion criteria $6 \mathrm{~h}$ after arrival at the ED, were transferred from other hospitals and did not meet the inclusion criteria on arrival at the ED, or were transferred directly from the ED to other hospitals. The institutional review board of each institution approved the study protocol, and informed consent was obtained from all patients before data collection. In this registry, information regarding the time of lactate measurement, blood culture, antibiotic administration, fluid administration, and use of vasopressors was recorded. A detailed description of the registry has been presented elsewhere [14-16].

In addition to the general septic shock registry described above, this study used an additional design. In our registry, overall cohorts were composed of patients who visited the ED directly or were transferred from another hospital. The cohort was limited to 
patients presenting directly to the EDs of the study institutions. We also only included patients who were enrolled for refractory hypotension because data regarding infusion of $30 \mathrm{~mL} / \mathrm{kg}$ of crystalloid fluid of patients enrolled for hypoperfusion were not investigated in our registry. Patients with missing information on any bundle component were excluded. Patients with missing information on their outcomes were also excluded.

\subsection{Definitions and Outcomes}

Patients were divided into 3 groups according to the interval from recognition of sepsis and septic shock to bundle achievement: group 1 ( $\leq 1 \mathrm{~h}$; reference), 2 (1-3 h) and 3 (3-6 h). We defined the recognition of septic shock as the time of refractory hypotension (persistent hypotension despite administration of fluid challenge).

If any of the five elements was not achieved within the specified time, bundle achievement was defined as failed. The outcomes of all groups were compared to those of the reference group. The decision to perform each element of the bundle was made by the treating physician. However, all the participating hospitals adhered to the recommendations of the SSC guidelines. The primary outcome of this study was in-hospital mortality; the secondary outcomes were 28-day and 90-day mortality.

\subsection{Statistical Analyses}

Continuous variables were analysed as means \pm standard deviation or medians with interquartile ranges, as appropriate, and categorical variables were analysed as absolute or relative frequencies. Continuous variables are presented by median (Q1-Q3) and tested by the Kruskal-Wallis test. The chi-squared test or Fisher's exact test were used for categorical variables.

The univariate and multivariable logistic regression analyses of each hour bundle achievement for predicting outcomes were conducted ( $<1-\mathrm{h}$ vs. $>1-\mathrm{h},<3-\mathrm{h}$ vs. $>3-\mathrm{h}$, and $<6$-h vs. $>6$-h, 2-group comparison). Multivariable logistic regression analysis was used to assess each bundle achievement group based on outcomes, with adjustment for confounding variables that were significant on univariate analysis. Variables yielding $p<0.2$ on univariate analysis were entered in a backward fashion in the multivariable analysis.

Patients were also divided into 3 groups according to the interval to bundle achievement, and outcomes were compared to examine the linear relationship in outcome for each group over time: group 1 ( $\leq 1 \mathrm{~h}$; reference), 2 (1-3 h), and 3 (3-6h). Comparisons of 2 groups with the reference group were analysed using a multivariable logistic model.

A two-sided $p$-value $<0.05$ was considered statistically significant, and the Bonferronicorrected threshold for statistical significance was computed and applied in each category. All statistical analyses were performed using SAS version 9.4 (SAS Institute; Cary, NC, USA) and R version 3.5.2 (R Foundation for Statistical Computing, Vienna, Austria).

\section{Results}

\subsection{Participant Characteristics}

A total of 1777 patients with refractory hypotension visited the ED directly during the study period. Of these, 165 patients with missing information on bundle achievement were excluded (Figure 1). Finally, 1612 patients were included. The number of patients in group 1,2 and 3 were 461, 637 and 293, respectively. The baseline characteristics of the patients in group 1, 2 and 3 are shown in Table 1. The mean patient age of group 1, 2 and 3 were 68,70 and 71 years, respectively. There was no significant difference in regard to age and male proportion. In-hospital mortality of group 1 was $13.8 \%(n=64)$. The median time from ED visit to shock recognition was $87 \mathrm{~min}$ (interquartile range, 26-150 $\mathrm{min}$ ). The 1-h bundle was achieved in 461 patients (28.6\%). The mean initial SBP was not significantly different between the group 1,2 and $3(p=0.152)$. The median lactate level between the group 1, 2 and 3 was significantly different ( 2.5 vs. 2.7 vs. $3.2 \mathrm{mmol} / \mathrm{L}, p<0.001)$. In-hospital mortality was not significantly different between 3 groups $(13.8 \%$ vs. $16.9 \%$ vs. $20.1 \%, p=0.075)$. Univariate logistic regression analysis of 1-, 3- and 6-h bundles (2 group 
comparison) was performed to predict in-hospital mortality (Table 2). Achievement of a 1-h bundle was significantly associated with lower in-hospital mortality $(p=0.005)$. The odds ratios (ORs) of 3- and 6-h bundle achievements for in-hospital mortality were significantly low (OR $=0.603$ and 0.511 , respectively; $p<0.001$ both). Old age, Sequential Organ Failure Assessment score, and Acute Physiologic Assessment and Chonic Health Evaluation score were associated with higher in-hospital mortality in the univariate analysis (Supplementary Table S1).

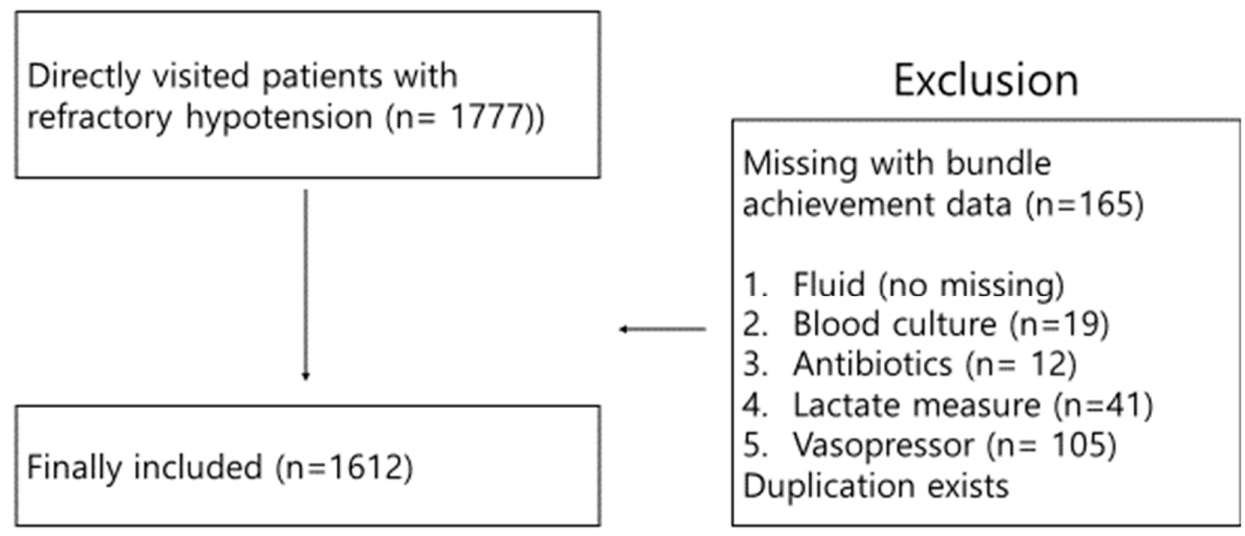

Figure 1. Patient selection flow diagram.

Table 1. Comparison of and clinical characteristics of the 3 bundle groups.

\begin{tabular}{|c|c|c|c|c|}
\hline Variables & $\begin{array}{c}\text { Group } 1 \\
(<1 \mathrm{~h}) \\
(n=461)\end{array}$ & $\begin{array}{c}\text { Group } 2 \\
(1-3 \mathrm{~h}) \\
(n=637)\end{array}$ & $\begin{array}{c}\text { Group } 3 \\
(3-6 \mathrm{~h}) \\
(n=293)\end{array}$ & $p$ Value \\
\hline Age, years & $68(60-76)$ & $70(59-78)$ & $71(60-78)$ & 0.063 \\
\hline Male, $n(\%)$ & $255(55.3)$ & $352(55.2)$ & $173(59)$ & 0.514 \\
\hline \multicolumn{5}{|l|}{ Initial vital signs } \\
\hline $\mathrm{SBP}, \mathrm{mm} \mathrm{Hg}$ & $91(77-111)$ & $89(74-108)$ & $88(74-107)$ & 0.152 \\
\hline $\mathrm{DBP}, \mathrm{mm} \mathrm{Hg}$ & $56(48-67)$ & $54(45-64)$ & $54(46-64)$ & 0.057 \\
\hline Heart rate, beat per min & $111(94-128)$ & $111(93-127)$ & $111(94-130)$ & 0.899 \\
\hline Respiratory rate, per min & $20(18-24)$ & $20(18-22)$ & $20(18-23)$ & 0.054 \\
\hline Body temperature, ${ }^{\circ} \mathrm{C}$ & $38.1(37.1-38.9)$ & $38(36.9-39)$ & $38(36.8-38.8)$ & 0.256 \\
\hline \multicolumn{5}{|l|}{ Comorbidities, $n(\%)$} \\
\hline Hypertension & $184(39.9)$ & $252(39.5)$ & $103(35.1)$ & 0.361 \\
\hline Diabetes mellitus & 105 (22.7) & $180(28.2)$ & $73(24.9)$ & 0.114 \\
\hline Cardiac disease & $81(17.5)$ & $72(11.3)$ & $33(11.2)$ & 0.005 \\
\hline COPD & $40(8.6)$ & $46(7.2)$ & $20(6.8)$ & 0.566 \\
\hline CKD & $33(7.1)$ & $47(7.3)$ & $17(5.8)$ & 0.668 \\
\hline Chronic liver disease & $57(12.3)$ & $68(10.6)$ & $25(8.5)$ & 0.253 \\
\hline \multicolumn{5}{|l|}{ Infection site, $n(\%)$} \\
\hline Respiratory & $123(26.6)$ & $128(20.1)$ & $66(22.5)$ & 0.036 \\
\hline Urinary tract & $93(20.1)$ & $140(21.9)$ & $51(17.4)$ & 0.271 \\
\hline Gastrointestinal tract & $48(10.4)$ & $104(16.3)$ & $37(12.6)$ & 0.016 \\
\hline Hepato-biliary and pancreas & 85 (18.4) & $96(15.1)$ & 57 (19.4) & 0.167 \\
\hline Others* & $20(4.3)$ & $32(5)$ & $18(6.1)$ & 0.543 \\
\hline Lactate, $\mathrm{mmol} / \mathrm{L}$ & $2.5(1.7-3.5)$ & $2.7(1.6-4.8)$ & $3.2(1.9-5)$ & $<.0001$ \\
\hline SOFA & $6(4-8)$ & $6(4-8)$ & $6(4-8)$ & 0.635 \\
\hline APACHE 2 & $19(13-25)$ & $19(13-25)$ & $21(15-27)$ & 0.021 \\
\hline Positive blood culture, $n(\%)$ & $208(45.1)$ & $289(45.3)$ & $135(46)$ & 0.966 \\
\hline \multicolumn{5}{|l|}{ Outcomes, $n(\%)$} \\
\hline In-hospital mortality & $64(13.8)$ & $108(16.9)$ & $59(20.1)$ & 0.075 \\
\hline $\begin{array}{l}\text { 28-day mortality } \\
\quad(n=1292)\end{array}$ & $57(13.2)$ & 98 (16.4) & 55 (20.6) & 0.037 \\
\hline $\begin{array}{c}90-\text { day mortality } \\
(n=1148)\end{array}$ & $102(26.1)$ & $138(26.4)$ & 75 (31.7) & 0.243 \\
\hline
\end{tabular}

APACHE 2: Acute Physiology and Chronic Health Evaluation 2, CKD: chronic kidney disease, COPD: chronic obstructive pulmonary disease, DBP: diastolic blood pressure, SOFA: Sequential Organ Failure Assessment. Continuous variables are presented by median (Q1-Q3) and tested by the Kruskal-Wallis test, and categorical variables are presented by $n(\%)$ and tested by the chi-squared test. ${ }^{*}$ Others includes soft tissue, central nervous system, catheter related, blood stream and endocarditis as infection site. 
Table 2. Univariate and multivariable logistic regression analysis for in-hospital mortality.

\begin{tabular}{ccccccc}
\hline Variables & Unadjusted OR & 95\% CI of OR & $\boldsymbol{p}$ Value & Adjusted OR & 95\% CI of OR & $p$ Value \\
\hline $\begin{array}{c}\text { Bundle achievement } \\
(2 \text { group comparison) }\end{array}$ & & & & & & \\
1-h bundle $(<1$ h vs. $>1$ h) & 0.649 & $0.481-0.877$ & 0.005 & 0.74 & $0.522-1.049$ & 0.091 \\
3-h bundle $(<3$ h vs. $>3$ h) & 0.603 & $0.465-0.783$ & $<0.001$ & 0.604 & $0.446-0.819$ & 0.001 \\
6-h bundle $(<6$ h vs. $>6$ h) & 0.511 & $0.369-0.707$ & $<0.001$ & 0.458 & $0.312-0.672$ & $<0.01$ \\
\hline
\end{tabular}

CI: confidence interval, OR: odds ratio.

3.2. Multivariate Logistic Regression Analysis of 1-, 3- and 6- $h$ Bundle Achievement to Predict in-Hospital Mortality (2 Group Comparison)

The adjusted OR of 1-h bundle achievement (<1 vs. $>1$ h, 2-group comparison) was 0.74 in predicting in-hospital mortality on multivariable logistic regression, but it was not significant (confidence interval [CI]: 0.522-1.049, $p=0.091$ ) (Table 2). Three-hour bundle achievement (<3 vs. $>3 \mathrm{~h}, 2$-group comparison) was significantly associated with lower in-hospital mortality (OR $=0.604, \mathrm{CI}: 0.446-0.819, p=0.001)$; 6-h bundle achievements were also associated with lower in-hospital mortality $(\mathrm{OR}=0.458, \mathrm{CI}: 0.312-0.672, p<0.01)$.

3.3. Comparison of Outcomes to Examine the Linear Relationship for 3 Groups over Time: Group 1 ( $\leq 1 \mathrm{~h}$; Reference), 2 (1-3 h), and 3 (3-6 h)

Multivariable analyses were performed to examine the effect of time delay in bundle achievement, with the 1-h bundle achievement group as the reference group. There were 461 patients in group 1 (reference group), and were 637 and 293 patients in groups 2 and 3, respectively. There was no significant difference or trend over time in in-hospital mortality of group 2 and 3 compared to group 1 on multivariable logistic regression analysis (Figure S1).

Multivariable analyses were conducted to predict the 28-day mortality of group 2 and 3 , with the group 1 as the reference group. There was no significant difference in the 28-day mortality between the 2, 3 groups and the group 1 on multivariable logistic regression analysis (Figure S2). There was no significant difference in the 90-day mortality between the groups (Figure S3).

\section{Discussion}

In this study, we found that the achievement of a 1-h bundle was not independently associated with improved outcomes in patients with septic shock in the ED. No linear association was observed between the time delay in bundle achievement and outcomes. However, 3- and 6-h bundle achievement were all associated with a better outcome.

To the best of our knowledge, this is the first study to address the impact of 1-h bundle achievement on the outcomes of patients with septic shock. Several studies have addressed individual elements of bundle treatment in sepsis or septic shock, but no study has investigated the impact of whole bundle achievement. Our study population was selected from a prospective, multicenter study with a large sample size. Our registry had all information regarding bundle elements, especially time variables, although it was not constructed to examine the impact of 1-h bundle achievement. There are controversies regarding the 1-h bundle treatment, and this study may be the basis for future research and may help clinicians in sepsis care.

The effectiveness of bundle therapy in sepsis and septic shock treatment is controversial [11,17-19]. In a multicenter retrospective cohort study, the group that failed to achieve severe sepsis and septic shock performance measure bundle (SEP-1) had a higher crude mortality than the group that achieved well, but no significant difference was found after adjusting for clinical variables and disease severity [20]. There were five elements in SEP-1, similar to those in the 1-h bundle, but the time limits were $3 \mathrm{~h}$ and $6 \mathrm{~h}$. In a study by Baghdadi et al., timely lactate measurement was associated with reduced mortality, but full SEP-1 adherence was not associated with improved outcome in patients with hospital- 
onset or community-onset sepsis [21]. According to one systematic review, no high- or moderate-level evidence showed that SEP-1 or its haemodynamic interventions improve the survival rate of adults with sepsis [22]. In a study analysing the effect of the 3-h bundle of sepsis care on 49,311 patients in 149 hospitals in New York, rapid bundle completion was associated with reduced in-hospital mortality [9]. A longer time to bundle completion was associated with higher rates of risk-adjusted in-hospital mortality. However, as the time to achieve bundle treatment was delayed, outcomes were not significantly different in our study despite the bundle achievement group showing significantly better outcomes than the failed group when comparing 2 groups (that is, 2-h achievement vs. 2-h failure). Our study consisted of five bundle treatment elements and the study in New York consisted of three elements (blood culture, lactate level measurement, and antibiotic administration); hence, comparison would be difficult.

Our study has several limitations. First, our data were not constructed to investigate 1-h bundle treatment. Our data were not part of a performance improvement initiative, as we performed secondary analysis of prospectively collected data. Performance improvement examining a 1-h bundle would be better to validate its prognostic value. Second, patients with hypoperfusion were not included in this study. Hence, these data are generalizable to the overtly hypotensive population with sepsis and not the cryptic shock population. Third, it is unclear whether there was a start of rapid administration of $30 \mathrm{~mL} / \mathrm{kg}$ crystalloid fluid within $1 \mathrm{~h}$ of shock recognition because we defined the time of shock recognition as the time of refractory hypotension despite fluid administration. It could be argued that our analysis did not consist of five bundle treatments and that it consisted of only four bundle treatments. However, it is likely that start of rapid fluid administration might be achieved within 1-h because the median time from ED triage to fluid completion was $87 \mathrm{~min}$. In the SSC guidelines, it is mandated that rapid fluid administration should be initiated within 1-h of shock recognition. We believe that start of fluid administration was performed as rapid as fast given median time. Fourth, the inherent limitations of a registry-based observational study should be acknowledged. Although our registry collected all variables related to bundle treatment elements, we cannot exclude the influence of unmeasured confounders that may have affected the results despite adjustment for the differences in the baseline risk factors with multivariable analysis. Finally, the differences in the outcomes of each participating hospital were not addressed.

\section{Conclusions}

The 1-h bundle was achieved by $28.6 \%$ of septic shock patients who were treated with a protocolized bundle therapy in the ED and 1-h bundle was not independently associated with better outcomes. No linear association was observed between the time delay in bundle achievement and patient outcome. However, 3- and 6-h bundle achievement were all associated with better outcomes compared to those who failed in comparison between the 2 groups. These data suggest that further investigation into the clinical implications of 1 -h bundle achievement in patients with septic shock is warranted.

Supplementary Materials: The following are available online at https:/ /www.mdpi.com/2077-03 83/10/3/527/s1, Table S1: Global univariate logistic regression analysis for in-hospital mortality; Figure S1: Adjusted odds ratios of each group for in-hospital mortality over time ( $\leq 1 \mathrm{~h}$; reference) in multivariable logistic regression analysis. Figure S2: Adjusted odds ratios of each group for 28-day mortality over time ( $\leq 1 \mathrm{~h}$; reference) in multivariable logistic regression analysis. Figure S3: Adjusted odds ratios of each group for 90-day mortality over time ( $\leq 1 \mathrm{~h}$; reference) in multivariable logistic regression analysis.

Author Contributions: B.S.K., W.Y.K. (Won Young Kim) and T.H.L. designed the study. S.-H.C., K.K., T.G.S., Y.H.J. and S.M.R. performed the data analysis. Y.S.P. and W.Y.K. (Woon Yong Kwon) contributed to data interpretation, writing, and approval of the final manuscript. H.S.C., S.P.C., G.J.S. and H.K. contributed to data collection, data interpretation, and editing of text. D.S. performed the statistical analysis. B.S.K., W.Y.K. and T.H.L. drafted the manuscript. All authors participated in 
the interpretation of the results. All authors have read and agreed to the published version of the manuscript.

Funding: This research was supported by the Bio and Medical Technology Development Program of the National Research Foundation (NRF) and was funded by the Korean government (MSIT) (No. NRF-2018R1C1B5043803).

Institutional Review Board Statement: This study was approved by the institutional review boards of each participating institute, and informed consent was obtained before data collection. (1) Asan Medical Center (S2015-1918-0002), (2) Gangnam Sacred Heart Hospital (2015-11-142), (3) Gangnam Severance Hospital (3-2015-0227), (4) Hanyang University Hospital (HYUH 2015-11-013-022), (5) Korea University Anam Hospital (HRPC2016-184), (6) Korea University Kuro Hospital (KUGH15358001), (7) Samsung Medical Center (SMC2015-09-057-057), (8) Seoul National University Hospital (J-1408-003-599), (9) Seoul National University Bundang Hospital (B-1409/266-401), (10) Severance Hospital (4-2015-0929), and (11) Seoul National University Boramae Hospital (IRB-16-2014-36).

Informed Consent Statement: Informed consent was obtained before data collection.

Data Availability Statement: Data available on request due to restrictions eg privacy or ethical.

Acknowledgments: We thank Yun Jin Kim in the Biostatistical Consulting and Research Lab, Hanyang University for assistance with the statistical analysis. We thank the Korean Shock Society (KoSS) Investigators.

Conflicts of Interest: The authors declare no conflict of interest.

\section{References}

1. Rudd, K.E.; Johnson, S.C.; Agesa, K.M.; Shackelford, K.A.; Tsoi, D.; Kievlan, D.R.; Colombara, D.V.; Ikuta, K.S.; Kissoon, N.; Finfer, S. Global, regional, and national sepsis incidence and mortality, 1990-2017: Analysis for the Global Burden of Disease Study. Lancet 2020, 395, 200-211. [CrossRef]

2. Singer, M.; Deutschman, C.S.; Seymour, C.W.; Shankar-Hari, M.; Annane, D.; Bauer, M.; Bellomo, R.; Bernard, G.R.; Chiche, J.-D.; Coopersmith, C.M. The third international consensus definitions for sepsis and septic shock (Sepsis-3). JAMA 2016, 315, 801-810. [CrossRef] [PubMed]

3. Harrison, D.A.; Welch, C.A.; Eddleston, J.M. The epidemiology of severe sepsis in England, Wales and Northern Ireland, 1996 to 2004: Secondary analysis of a high quality clinical database, the ICNARC Case Mix Programme Database. Crit. Care 2006, 10, R42. [CrossRef] [PubMed]

4. Kaukonen, K.-M.; Bailey, M.; Suzuki, S.; Pilcher, D.; Bellomo, R. Mortality related to severe sepsis and septic shock among critically ill patients in Australia and New Zealand, 2000-2012. JAMA 2014, 311, 1308-1316. [CrossRef]

5. $\quad$ Rhee, C.; Gohil, S.; Klompas, M. Regulatory mandates for sepsis care-reasons for caution. N. Engl. J. Med. 2014, 370, 1673-1676. [CrossRef]

6. Rhodes, A.; Evans, L.E.; Alhazzani, W.; Levy, M.M.; Antonelli, M.; Ferrer, R.; Kumar, A.; Sevransky, J.E.; Sprung, C.L.; Nunnally, M.E. Surviving sepsis campaign: International guidelines for management of sepsis and septic shock: 2016. Intensive Care Med. 2017, 43, 304-377. [CrossRef]

7. Levy, M.M.; Evans, L.E.; Rhodes, A. The surviving sepsis campaign bundle: 2018 update. Intensive Care Med. 2018, 44, 925-928. [CrossRef]

8. SCCM. Surviving Sepsis Campaign/Guidelines/Adult Patients. Available online: https://www.sccm.org/SurvivingSepsisCamp aign/Guidelines/Adult-Patients (accessed on 10 October 2019).

9. Seymour, C.W.; Gesten, F.; Prescott, H.C.; Friedrich, M.E.; Iwashyna, T.J.; Phillips, G.S.; Lemeshow, S.; Osborn, T.; Terry, K.M.; Levy, M.M. Time to treatment and mortality during mandated emergency care for sepsis. N. Engl. J. Med. 2017, 376, 2235-2244. [CrossRef]

10. Marik, P.E.; Farkas, J.D.; Spiegel, R.; Weingart, S. Rebuttal From Drs Marik, Farkas, Spiegel et al. Chest 2019, 155, 17. [CrossRef]

11. Marik, P.E.; Farkas, J.D.; Spiegel, R.; Weingart, S.; Aberegg, S.; Beck-Esmay, J.; Carroll, S.; Kenny, J.-E.; Koyfman, A.; Lin, M. POINT: Should the Surviving Sepsis Campaign guidelines be retired? Yes. Chest 2019, 155, 12-14. [CrossRef]

12. Mouncey, P.R.; Osborn, T.M.; Power, G.S.; Harrison, D.A.; Sadique, M.Z.; Grieve, R.D.; Jahan, R.; Harvey, S.E.; Bell, D.; Bion, J.F. Trial of early, goal-directed resuscitation for septic shock. N. Engl. J. Med. 2015, 372, 1301-1311. [CrossRef] [PubMed]

13. Investigators, P. A randomized trial of protocol-based care for early septic shock. N. Engl. J. Med. 2014, 370, 1683-1693. [CrossRef] [PubMed]

14. Ko, B.S.; Kim, K.; Choi, S.-H.; Kang, G.H.; Shin, T.G.; Jo, Y.H.; Ryoo, S.M.; Beom, J.H.; Kwon, W.Y.; Han, K.S. Prognosis of patients excluded by the definition of septic shock based on their lactate levels after initial fluid resuscitation: A prospective multi-center observational study. Crit. Care 2018, 22, 47. [CrossRef] [PubMed]

15. Jung, S.M.; Kim, Y.-J.; Ryoo, S.M.; Kim, W.Y. Relationship between low hemoglobin levels and mortality in patients with septic shock. Acute Crit. Care 2019, 34, 141. [CrossRef] 
16. Ryoo, S.M.; Han, K.S.; Ahn, S.; Shin, T.G.; Hwang, S.Y.; Chung, S.P.; Hwang, Y.J.; Park, Y.S.; Jo, Y.H.; Chang, H.L. The usefulness of C-reactive protein and procalcitonin to predict prognosis in septic shock patients: A multicenter prospective registry-based observational study. Sci. Rep. 2019, 9, 1-8. [CrossRef]

17. Levy, M.M.; Rhodes, A.; Evans, L.E.; Antonelli, M.; Bailey, H.; Kesecioglu, J.; Zimmerman, J.J.; Beale, R.; De Backer, D.; Roca, R.F. COUNTERPOINT: Should the Surviving Sepsis Campaign guidelines be retired? No. Chest 2019, 155, 14-17. [CrossRef]

18. Freund, Y.; Khoury, A.; Möckel, M.; Karamercan, M.; Dodt, C.; Leach, R.; Bloom, B.; Garcia-Castrillo, L. European Society of Emergency Medicine position paper on the 1-h sepsis bundle of the Surviving Sepsis Campaign: Expression of concern. Eur. J. Emerg. Med. 2019, 26, 232-233. [CrossRef]

19. Murri, R.; Taccari, F.; Palazzolo, C.; Fantoni, M.; Cauda, R. IDSA did not endorse the Surviving Sepsis Campaign guidelines. Clin. Infect. Dis. 2018, 66, 1815-1816. [CrossRef]

20. Rhee, C.; Filbin, M.; Massaro, A.F.; Bulger, A.; McEachern, D.; Tobin, K.A.; Kitch, B.; Thurlo-Walsh, B.; Kadar, A.; Koffman, A. Compliance with the national SEP-1 quality measure and association with sepsis outcomes: A multicenter retrospective cohort study. Crit. Care Med. 2018, 46, 1585. [CrossRef]

21. Baghdadi, J.D.; Brook, R.H.; Uslan, D.Z.; Needleman, J.; Bell, D.S.; Cunningham, W.E.; Wong, M.D. Association of a Care Bundle for Early Sepsis Management with Mortality Among Patients With Hospital-Onset or Community-Onset Sepsis. JAMA Intern. Med. 2020, 180, 707-716. [CrossRef]

22. Pepper, D.J.; Jaswal, D.; Sun, J.; Welsh, J.; Natanson, C.; Eichacker, P.Q. Evidence Underpinning the Centers for Medicare \& Medicaid Services' Severe Sepsis and Septic Shock Management Bundle (SEP-1) A Systematic Review. Ann. Intern. Med. 2018, 168, 558-568. [PubMed] 